\title{
Rediseño de Módulo Didáctico de Levitación Magnética
}

\author{
Redisign of Didactic Module of Magnetic Levitation
}

\author{
Saida Charre-Ibarra ${ }^{a}$, Mario Rodríguez-Díaz ${ }^{b}$, Jorge Gudiño-Lau $^{c}$ Janeth Alcalá-Rodriguez $^{c}$, \\ Daniel Vélez-Díaz ${ }^{d}$
}

\begin{abstract}
:
This paper shows the redesign of a training module of magnetic levitation in closed architecture, developing the subsystems for the sensing stage, power stage and structure. The objective of the redesign is to have a training module that allows the application of different control techniques; and thus determine the best strategies for the control of magnetic levitation systems.

The results are validated experimenting with the module and data is collected for both the inputs and the output of the system in open architecture.
\end{abstract}

\section{Keywords:}

Closed architecture, open architecture, magnetic levitation, infrared sensor, open loop control

\section{Resumen:}

Este artículo muestra el rediseño de un módulo didáctico de levitación magnética en arquitectura cerrada, desarrollando los subsistemas para la etapa de sensado, etapa de potencia y estructura. El objetivo del rediseño es contar con un módulo didáctico que permita la aplicación de diferentes técnicas de control; y así determinar las mejores estrategias para el control de sistemas que funcionan con levitación magnética.

Se validan los resultados experimentando con el módulo y registrando las variables de entrada y salida del sistema en arquitectura abierta.

\section{Palabras Clave:}

Arquitectura cerrada, arquitectura abierta, levitación magnética, sensor infrarrojo, control en lazo abierto

\section{Introducción}

La levitación magnética (Maglev) es un fenómeno muy interesante en el que un objeto se mantiene en el aire con la aplicación de una fuerza magnética que actúa en contra de la atracción gravitacional. Este proceso tiene dos fases; una es posicionar el objeto en la posición deseada y la otra es mantener el objeto en esa posición deseada (Anirudh et al., 2015). Este fenómeno tiene muchas aplicaciones prácticas, principalmente en: el sistema de transporte (por ejemplo, tren de pasajeros de alta velocidad), la suspensión de modelos de túnel de viento (Kumar y Minz, 2016), (Perren,2003), sistemas mecánicos de almacenamiento de energía (Clark,
Thompson y Thornton, 1998) y levitación de microrobots en tres dimensiones (Khamesee, Kato y Nakamura, 2003).

El avance de la tecnología a nivel mundial en el área de la levitación magnética, obliga a las instituciones educativas a proporcionar una mejor preparación a los estudiantes, principalmente de las áreas de las tecnologías e ingenierías, donde se imparten cursos de control automático y se puede introducir el control de energía magnética, para posicionar y mantener un objeto. Existen diversos prototipos educacionales, donde la importancia de estos radica en el bajo costo que estos tienen con relación a sistemas reales (Moriano y Naranjo, 2012). Actualmente en la Facultad de Ingeniería Electromecánica de la Universidad de Colima se cuenta

\footnotetext{
a Autor de Correspondencia, Universidad de Colima., Facultad de Ingeniería Electromecánica. Email: scharre@ucol.mx

b Alumno de la Facultad de Ingeniería Electromecánica, de la Universidad Colima. Hibari9191@ gmail.com

${ }^{\text {c }}$ Profesor Investigador de la Universidad de Colima, de la Facultad de Ingeniería Electromecánica. Jorge Gudiño Lau jglau@ucol.mx ; Janeth 
con un módulo didáctico de levitación magnética que se encuentra en arquitectura cerrada creado por la empresa De Ville International S.A. de C.V.; por lo que se plantea su rediseño, de forma que el módulo permita experimentar con diferentes técnicas de control, lo cual implica el diseño e integración de varios subsistemas, como la estructura, la electrónica para el sensado, la electrónica de potencia y de control.

\section{Descripción del Módulo en Arquitectura cerrada}

En la Figura 1 se presenta el módulo didáctico Maglev trainer, el cual se compone principalmente de los elementos físicos listados en la Tabla I.

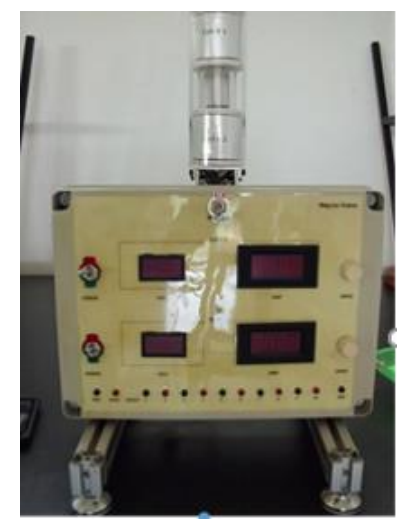

Fig. 1. Maglev trainer (DE VILLE INTERNATIONAL S.A. DE C.V).

Tabla I Elementos del módulo Maglev trainer.

\begin{tabular}{|c|c|c|}
\hline Nombre & Cantidad & Función \\
\hline Bobinas & 2 & $\begin{array}{l}\text { Crear un campo } \\
\text { magnético. }\end{array}$ \\
\hline Switch & 3 & $\begin{array}{l}1 \text { Función encendido y } \\
\text { apagado } \\
2 \text { Función de atracción o } \\
\text { repulsión. }\end{array}$ \\
\hline $\begin{array}{l}\text { Potenció } \\
\text { metros }\end{array}$ & 2 & Regulación de corriente. \\
\hline Display & 4 & $\begin{array}{l}2 \text { Voltímetros y } 2 \\
\text { amperímetros. }\end{array}$ \\
\hline
\end{tabular}

Su diagrama de bloques en arquitectura cerrada se muestra en la Figura 2, donde se observan los pasos de un ciclo de trabajo manual, inicia con un voltaje a $127 \mathrm{~V}$ en corriente alterna (AC) hacía los transformadores reductores de $127 \mathrm{~V}-15 \mathrm{~V}$ a $10 \mathrm{~A}$, este voltaje pasa directamente al triac BT136, con ayuda de un potenciómetro este voltaje es regulado, la salida obtenida puede ingresar a cualquiera de los dos embobinados debido al multi-switch que tiene integrado a la salida, de forma que el voltaje de entrada de los embobinados sea controlado y el efecto electromagnético sobre un imán cerámico genere la levitación o repulsión.

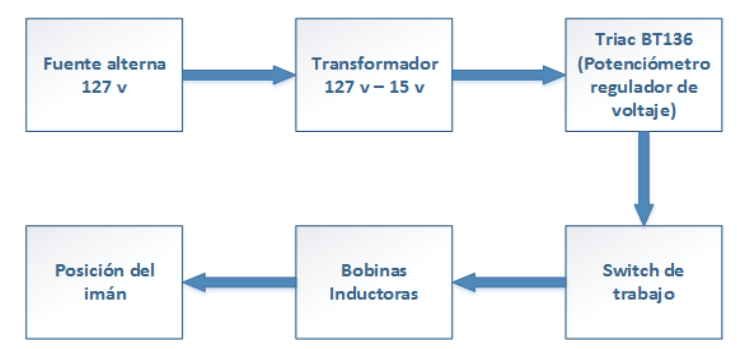

Fig. 2. Diagrama a bloques del módulo en arquitectura cerrada

En base a la estructura de un sistema de control en lazo abierto (Figura 3), se identificaron los elementos de control en el módulo didáctico, Tabla II.

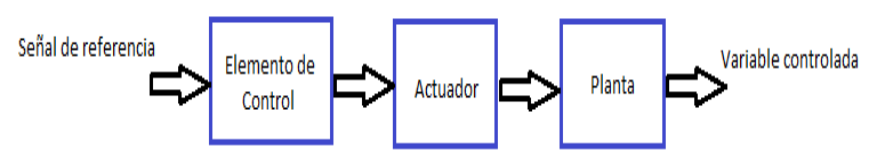

Fig. 3. Diagrama a bloques de un sistema de control en lazo abir.

Tabla I Elementos del sistema de control en lazo abierto

\begin{tabular}{|c|c|}
\hline Variable controlada & $\begin{array}{l}\text { Posición del imán } \\
\text { cerámico }\end{array}$ \\
\hline Señal de referencia & Voltaje y corriente \\
\hline Comparador & Regla \\
\hline Señal del error & - \\
\hline Controlador & Control manual \\
\hline Planta & Campo electromagnético \\
\hline Dispositivo de medición & - \\
\hline
\end{tabular}

El circuito del módulo es el que se muestra en la Figura 4 , en el que la alimentación de $127 \mathrm{~V}$ entra directamente a los transformadores para bajar el voltaje a $15 \mathrm{~V}$, después los puentes de diodos MB362 rectifican el voltaje, este voltaje entra al triac BT136 y la salida del mismo se dirige a los switches de trabajo que van conectados a las bobinas de atracción o repulsión. De la salida de este circuito también se alimentan los voltímetros y amperímetros. 


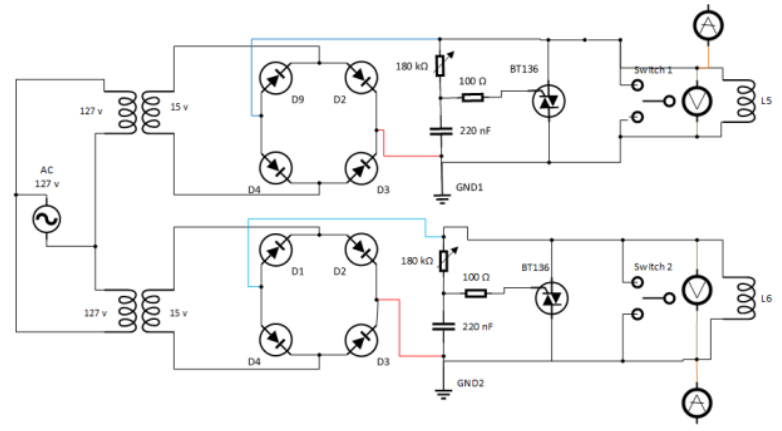

Fig. 4. Diagrama eléctrico en arquitectura cerrada

\section{Sistema de Potencia}

El rediseño del módulo en arquitectura abierta permitirá el monitoreo de las variables y la experimentación con diferentes estrategias de control tales como controles PID, on-off, inteligentes, en cascada, adaptables, entre otras. En la Figura 5 se presenta el diagrama a bloques del rediseño, donde se utiliza el Arduino Mega 2560 para el envío de la señal de control a la etapa de potencia implementada mediante un puente $\mathrm{H}$ (Monster motor shield), quien proporciona el voltaje y la corriente necesario a las bobinas para posicionar el imán.

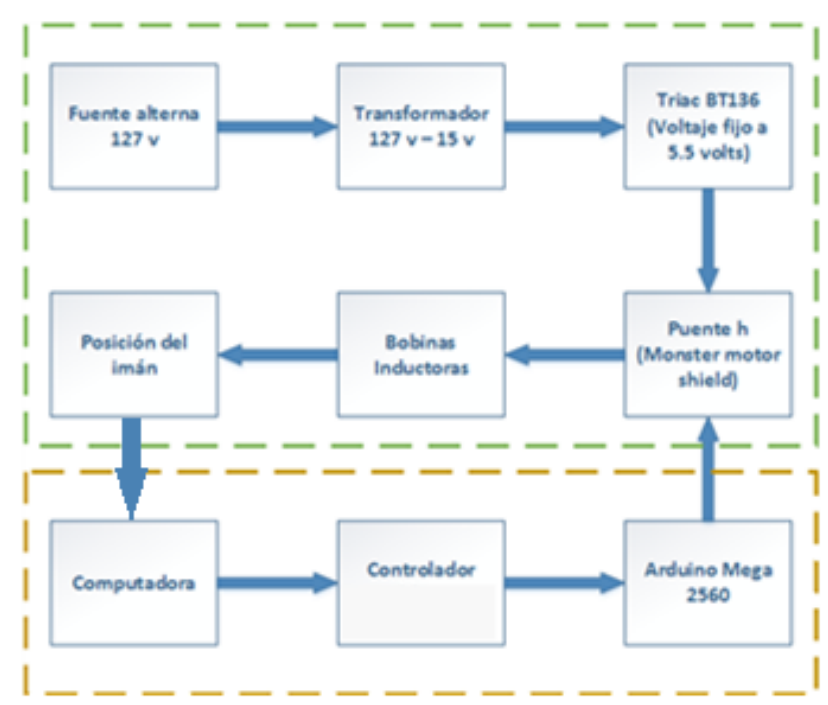

Fig. 5. Diagrama a bloques del módulo en arquitectura abierta.

Como parte de la etapa de potencia se anexo un puente $\mathrm{H}$, encargado de regular la corriente de trabajo del módulo, Figura 6.
Fig. 6. Puente H Monster Shield

El Puente H
con un par de
de puente
VNH2SP30
características
la tabla III.

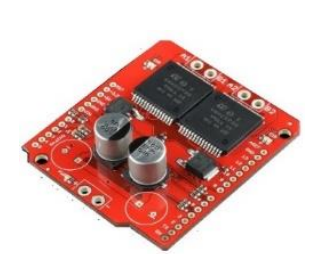

"Monster Shield" drivers de motor completo cuenta con las que se listan en

Tabla III Características del Monster Shield

\begin{tabular}{|ll|}
\hline Características & \\
\hline Voltaje máximo & $16 \mathrm{~V}$ \\
$\begin{array}{l}\text { Corriente continua } \\
\text { Máxima frecuencia de } \\
\text { trabajo }\end{array}$ & $14 \mathrm{~A}$ \\
\hline
\end{tabular}

El diagrama eléctrico del módulo se presenta en la Figura 7 , donde se muestra con un rectángulo azul las conexiones de control en el Arduino y el puente.

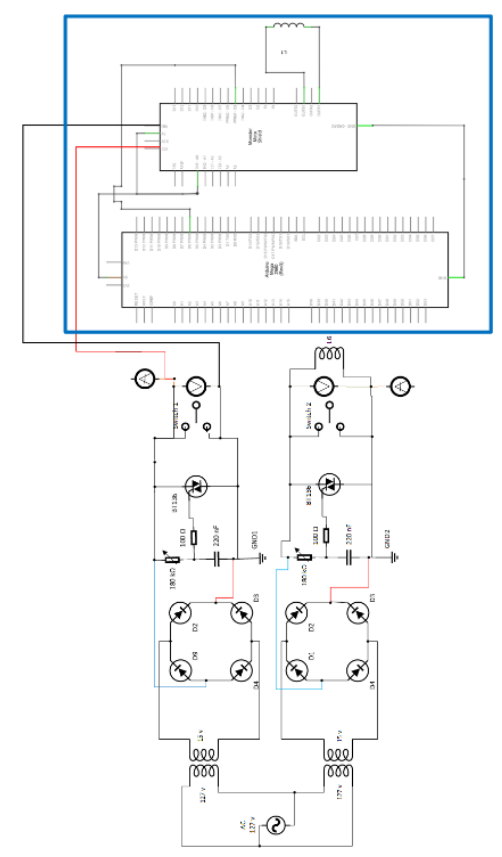

Fig. 7. Diagrama eléctrico en arquitectura abierta.

Para ubicar la posición del imán se coloco el sensor óptico reflectivo infrarrojo TCRT5000L, que cuenta con un campo de trabajo de $15 \mathrm{~mm}$ (Figura 8). Con la finalidad de hacer más visible la posición del imán dentro del cilindro se colocó una etiqueta con escala en milímetros. 


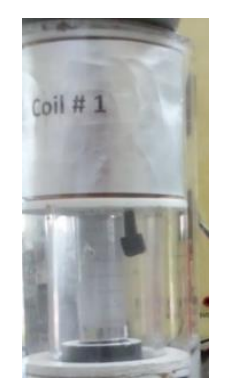

Fig. 8. Ubicación del sensor TCRT5000L

Se construyó un bloque de conexiones con la finalidad de poder obtener las variables y aplicar señales de control al módulo didáctico (Figura 9).

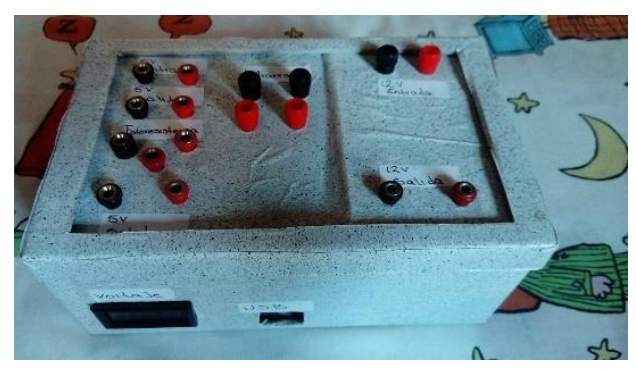

Fig. 9. Bloque de conexiones.

\section{Resultados}

En la figura 10 se presenta el módulo didáctico de levitación magnética en arquitectura abierta, con el bloque de conexiones, el cual posee un voltímetro correspondiente a la salida de voltaje del puente $\mathrm{H}$ que se aplica el módulo.

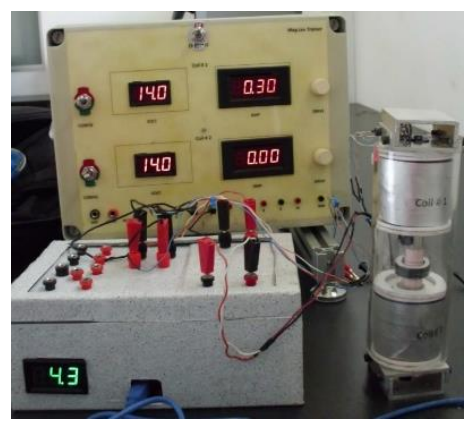

Fig. 10. Módulo didáctico de levitación magnética.

La Tabla IV muestra la relación de la corriente de trabajo respecto al pwm obtenido por el Arduino en un rango de 0 a 255 , siendo 0 un $0 \%$ y 255 un $100 \%$ del ciclo de trabajo, así como las lecturas obtenidas por el sensor infrarrojo correspondientes a las diferentes alturas que el imán cerámico alcanzo.

Tabla IV Relación de voltaje-corriente-posición del módulo didáctico

\begin{tabular}{lllll}
\hline $\begin{array}{c}\text { Voltaje } \\
\text { del } \\
\text { módulo }\end{array}$ & $\begin{array}{l}\text { Corriente } \\
\text { del Módulo }\end{array}$ & $\begin{array}{l}\text { Sensor } \\
\text { infrarrojo }\end{array}$ & Altura & Pwm \\
\hline 0 & 0 & $2.52 \mathrm{~V}$ & $0 \mathrm{~mm}$ & 30 \\
3 & $490 \mathrm{~mA}$ & $2.54 \mathrm{~V}$ & $1 \mathrm{~mm}$ & 40 \\
3.5 & $570 \mathrm{~mA}$ & $2.57 \mathrm{~V}$ & $2 \mathrm{~mm}$ & 42 \\
3.8 & $610 \mathrm{~mA}$ & $2.61 \mathrm{~V}$ & $3 \mathrm{~mm}$ & 45 \\
4 & $650 \mathrm{~mA}$ & $2.623 \mathrm{~V}$ & $4 \mathrm{~mm}$ & 50 \\
5 & $810 \mathrm{~mA}$ & $2.627 \mathrm{~V}$ & $5 \mathrm{~mm}$ & 54 \\
6 & $980 \mathrm{~mA}$ & $2.65 \mathrm{~V}$ & $6 \mathrm{~mm}$ & 60 \\
7 & $1.13 \mathrm{~A}$ & $2.66 \mathrm{~V}$ & $7 \mathrm{~mm}$ & 62 \\
8 & $1.32 \mathrm{~A}$ & $2.681 \mathrm{~V}$ & $8 \mathrm{~mm}$ & 70 \\
8.5 & $1.37 \mathrm{~A}$ & $2.668 \mathrm{~V}$ & $9 \mathrm{~mm}$ & 75 \\
8.7 & $1.41 \mathrm{~A}$ & $2.72 \mathrm{~V}$ & $10 \mathrm{~mm}$ & 80 \\
9 & $1.47 \mathrm{~A}$ & $2.74 \mathrm{~V}$ & $11 \mathrm{~mm}$ & 95 \\
10 & $1.63 \mathrm{~A}$ & $2.77 \mathrm{~V}$ & $12 \mathrm{~mm}$ & 120 \\
\hline
\end{tabular}

\section{Conclusiones}

Partiendo de un diseño funcional y probado, se rediseño en arquitectura abierta el módulo didáctico de levitación magnética Maglev trainer, con la finalidad de desarrollar prácticas y proyectos de investigación en el área de control de energía magnética. El sistema presentado contribuye a desarrollar conocimientos en nuevas tecnologías enfocadas a diferentes disciplinas tales como electrónica, control y máquinas eléctricas.

Con la finalidad de demostrar el funcionamiento del rediseño se presentó una tabla que muestra diferentes lecturas tomadas del módulo, aplicándole una señal externa de voltaje, observándose que se logró la correcta integración de los subsistemas diseñados para obtener un módulo en arquitectura abierta, incrementando su funcionalidad y permitiendo la sustitución de sus componentes en caso de ser necesario.

\section{Agradecimientos}

Los autores agradecen el apoyo a la Facultad de Ingeniería Electromecánica de la Universidad de Colima.

\section{Referencias}

[1] Anirudh, N., Jagannath, S., Saurabh, C., \& Binoy, R. (2015). Magnetic Ball Levitation System Control Using Sliding Mode Control and Fuzzy PD+I Control: A Comparative Study. DOI: 10.1109/EPETSG.2015.7510165.

[2] Pawan Kumar, P. \& Minz, S. (2016). Control of Magnetic Levitation System Using PD and PID controller. International Journal of Scientific Research and Education, ISSN-e 2321-7545, Vol. 4, No. 8, 5596-5602.

[3] Perren, G. (2003). Estudio de las aplicaciones prácticas de la levitación magnética (trenes maglev). Disponible en: https://www.fceia.unr.edu.ar/ fisica3/MagLev.pdf 